\title{
Multiphoton photoemission of gold nanopillars fabricated by carbon nanotube templates
}

\author{
Mahta Monshipouri \\ Nanophysics Research Lab, Department of Physics, University of Tehran, Tehran 1439955961, Iran and \\ Laboratory for Micro- and Nanotechnology, Paul Scherrer Institute, CH-5232 Villigen-PSI, Switzerland \\ Yaser Abdi ${ }^{\mathrm{a})}$ \\ Nanophysics Research Lab, Department of Physics, University of Tehran, Tehran 1439955961, Iran \\ Sara Darbari \\ ECE Department, Tarbiat Modares University, Tehran 111-14115, Iran \\ Soichiro Tsujino \\ Laboratory for Micro- and Nanotechnology, Paul Scherrer Institute, CH-5232 Villigen-PSI, Switzerland
}

(Received 7 December 2016; accepted 3 March 2017; published 20 March 2017)

\begin{abstract}
High-aspect ratio metallic nanopillars are promising as electron emitters in frequency ranges of terahertz and higher. However, producing nanopillars with aspect ratio of more than a factor of 10 by lithographic methods is still a challenge. Here, the authors report the fabrication of Au nanopillars using carbon nanotube forests as templates and a study on their near infrared photocurrent characterization. The pillars had the diameter of $100 \mathrm{~nm}$ and the aspect ratio up to 28. The authors observed an increase in the photocurrent from Au nanopillars with the increase in the nanopillar height. The observed photocurrent is compatible with the multiphoton photoemission and is uncorrelated to the pillar density. The results indicate that our proposed method is promising to produce electron sources for high frequency vacuum nanoelectronic devices. (C) 2017 American Vacuum Society. [http://dx.doi.org/10.1116/1.4978655]
\end{abstract}

\section{INTRODUCTION}

Carbon nanotubes (CNTs) have been actively studied for field emission applications, ${ }^{1-5}$ since their high aspect ratio enhances the electrostatic field at the nanotube tip apex and makes CNTs efficient field emitters at a low applied electric field. CNTs have been also studied intensively for high frequency electron source applications. ${ }^{6}$ This is because the field emission of electrons from solid surfaces relies on the quantum mechanical tunneling through the surface barriers, which has no apparent high frequency cut-off at terahertz (THz) and optical frequency range. We believe that coating CNTs with metals is advantageous for enhancement of high frequency response of CNT electron emitters in $\mathrm{THz}$ and optical frequencies, especially when metals such as $\mathrm{Au}$ or Ag with the low optical loss and with the higher plasmon frequency than CNTs are used. Since it is possible to grow long CNTs (Refs. 1-3) using the plasma-enhanced chemical vapor deposition (PECVD) method, metal nanopillars fabricated by the metal coating of long CNTs as the template can have the aspect ratios much higher than the values achievable by lithographic ${ }^{7,8}$ and electrochemical ${ }^{9,10}$ methods. Fabrication of nanorods lying on the substrates with the aspect ratio up to 25 was reported by using seeded growth ${ }^{11-13}$ and template-assisted method. ${ }^{14}$ However, to extract field emission current efficiently, upright and threedimensional architecture is preferred.

In this work, we therefore propose a method for fabrication of high-aspect-ratio $\mathrm{Au}$ nanopillars using CNT templates. Using numerical simulation, we studied field

a)Electronic mail: y.abdi@ut.ac.ir enhancement of the structures at $\mathrm{THz}$ and optical frequencies. We further studied the near infrared photocurrent of the fabricated Au nanopillars experimentally.

\section{EXPERIMENT}

\section{A. Au nanopillar fabrication using CNT templates}

In order to fabricate the CNT template, we used the PECVD method as shown in Fig. 1. We used nickel nanoislands as the catalyst for the PECVD growth of the CNTs. To prepare the nickel nanoislands, we annealed a 10-nm-thick nickel thin film deposited on a (100)-oriented p-type Si substrate [Fig. 1(a)] at $700^{\circ} \mathrm{C}$ in the presence of hydrogen gas for $30 \mathrm{~min}$ [Fig. 1(b)], and then we applied DC hydrogen plasma at the same temperature for 7 min [Fig. 1(c)] with the plasma power density of $5.2 \mathrm{~W} / \mathrm{cm}^{2}$. By adjusting the annealing temperature, we controlled the diameter of the nickel nanoislands. ${ }^{1}$ The island density was controlled by adjusting the plasma power density. To grow the CNTs, we introduced a mixture of $\mathrm{H}_{2}$ and acetylene gases [Figs. 1(d) and 1(e)] with the $\mathrm{H}_{2} / \mathrm{C}_{2} \mathrm{H}_{2}$ ratio of $100 / 45$ into the chamber and applied the plasma with the power density nominally equal to $7 \mathrm{~W} / \mathrm{cm}^{2}$ at the same substrate temperature. The CNT heights were controlled from $\sim 700 \mathrm{~nm}$ to $\sim 2.7 \mu \mathrm{m}$ by varying the growth time.

Finally, we deposited gold on the CNTs [Fig. 1(f)] by the DC magnetron sputtering method with a custom built sputtering instrument. The key factors to control the nanoparticle sizes and the coverage over CNTs were the flow rate of $\mathrm{Ar}$ gas, the plasma power, and the deposition time. We have optimized the conditions to coat the CNTs with a thin and 


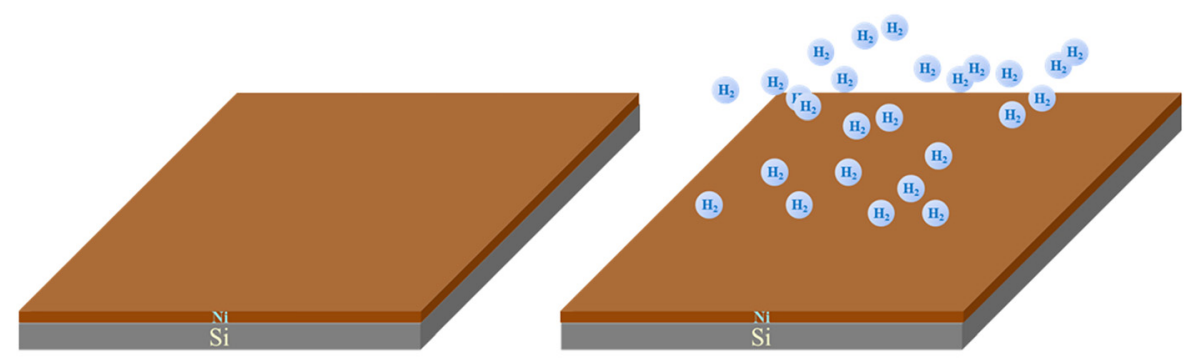

(a)

(b)

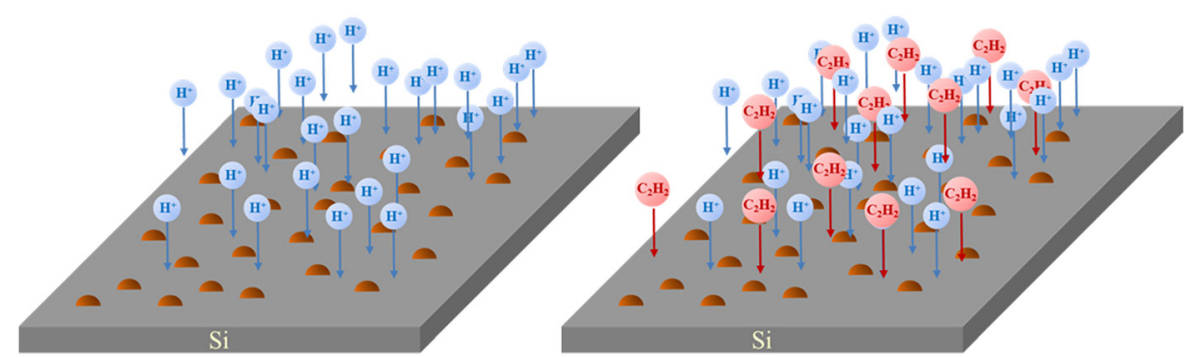

(c)

(d)

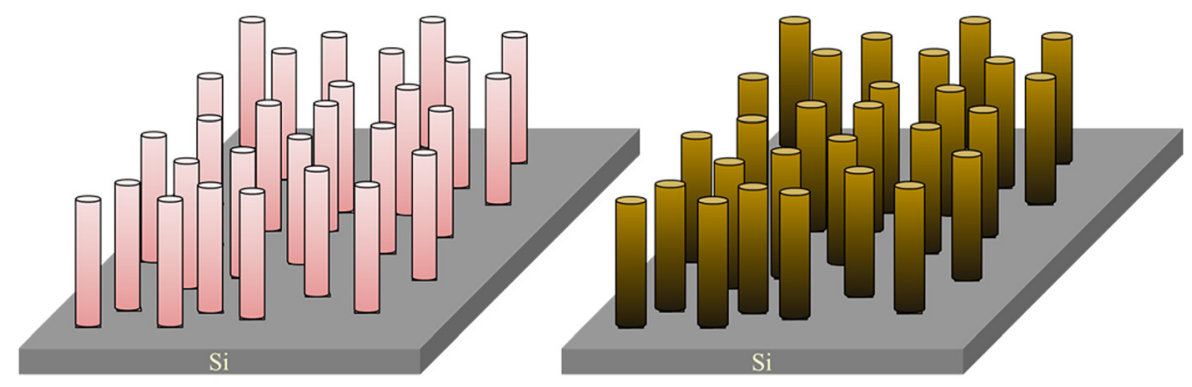

(e)

(f)

FIG. 1. (Color online) Fabrication steps for fabrication of the Au nanopillars using CNT templates: (a) deposition of Ni film, (b) annealing, (c) Ni nanoislands formation, (d) introducing acetylene gas, (e) growth of CNT forests, and (f) sputtering deposition of gold layer.

conformal Au layer. This was achieved with the Ar flow rate of $50 \mathrm{sccm}$ and the application of the plasma power of $180 \mathrm{~W}$ for $15 \mathrm{~min}$. We adjusted the plasma power by the plasma current when the applied voltage was approximately $4.5 \mathrm{kV}$. The Au-target and the counter electrode of our equipment were approximately separated by $20 \mathrm{~mm}$.

In the SEM pictures shown in Fig. 2, we compare the images of the grown CNTs [Fig. 2(a); after the step, Fig. 1(e)] and the completed Au nanopillars [Fig. 2(b); after the step, Fig. 1(f)]. By analyzing the SEM images, we found that the diameter of the CNTs was $\sim 70 \mathrm{~nm}$ and the diameter of the Au nanopillars was $\sim 100 \mathrm{~nm}$. This shows that the CNTs were homogeneously coated by the gold with the thickness of $\sim 15 \mathrm{~nm}$. Close inspection of the SEM images indicates that the gold layer consists of small gold particles with the diameters in the range of 5-10 nm, i.e., smaller than the estimated gold thickness.

Using this method, we prepared five $\mathrm{Au}$ nanopillar samples, samples A-E, with different pillar height and distributed pillar density as summarized in Table I. The
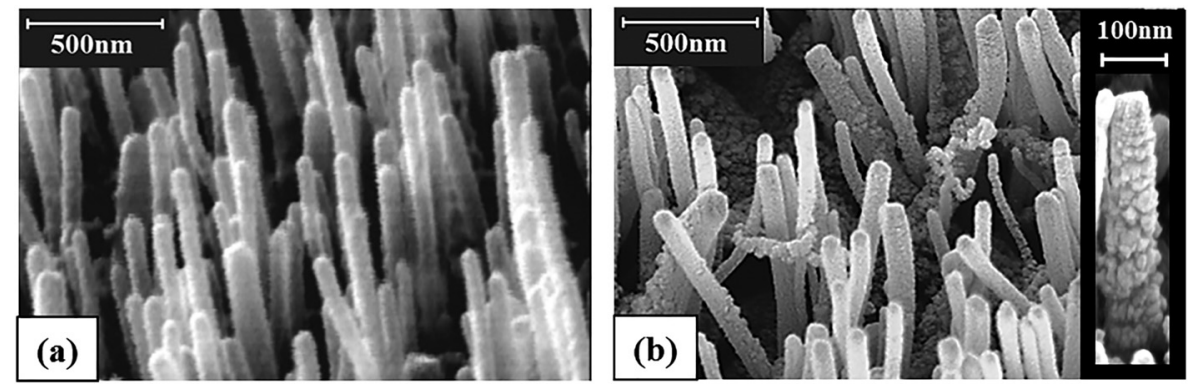

FIG. 2. SEM images of (a) as-grown CNTs, and (b) CNTs coated with gold. Inset shows a magnified image of the nanopillar's surface. 
TABLE I. Geometrical details of prepared samples.

\begin{tabular}{lcc}
\hline \hline Sample & Pillar height $(\mu \mathrm{m})$ & Pillar density $\left(\mu \mathrm{m}^{-2}\right)$ \\
\hline A & $0.7 \pm 0.17$ & 5.9 \\
B & $1.16 \pm 0.25$ & 8.0 \\
C & $1.05 \pm 0.15$ & 2.6 \\
D & $1.90 \pm 0.31$ & 1.4 \\
E & $2.70 \pm 0.12$ & 10.6 \\
\hline \hline
\end{tabular}

approximate pillar diameter for these samples was $100 \mathrm{~nm}$. We fabricated the Au nanopillars within a circular area with the diameter of $\sim 5 \mathrm{~mm}$ on the $\mathrm{Si}$ wafers by defining the nickel deposition area using photolithography after Ni deposition [Fig. 1(a)].

\section{B. Photoemission experiment}

To explore the impact of the aspect ratio of Au nanopillar in different samples, we studied the near infrared photocurrent. The schematic of the experimental setup is shown in Fig. 3. To produce near infrared light pulses, we used a mode-locked Ti:Sapphire oscillator with the nominal pulse duration of $10 \mathrm{fs}$, the pulse repetition time of $13.4 \mathrm{~ns}$, the optical bandwidth in the range of 750-850 nm, and the pulse energy of $\sim 1 \mathrm{~nJ}$. The pulse duration was increased to $13 \mathrm{fs}$ after passing through the window of the measurement chamber because of dispersion. The laser pulses with incident angle of $60^{\circ}$ with respect to the substrate normal direction were focused on the sample to a spot size of $\sim 50 \times 100 \mu \mathrm{m}^{2}$ and scanned across the sample. The generated photocurrent was collected by an anode biased at $1 \mathrm{kV}$ and separated from the sample by $\sim 7 \mathrm{~mm}$ by using a source-measure unit (Keithley 237). In order to study the polarization dependence of the photocurrent, we used a half-waveplate. As the insertion of the wave plate increased the pulse duration from 13 fs to $\sim 16 \mathrm{fs}$, we observed that the photocurrent signal decreased significantly. This was compatible with the multiphoton photoemission (see below). The typical pressure of the measurement chamber was below $5 \times 10^{-9}$ mbar.

\section{MODELING}

Using a finite-element electromagnetic wave simulator (COMSOL Multiphysics), we calculated the threedimensional electric field distribution of the Au nanopillars illuminated by $\mathrm{THz}$ and optical frequency electromagnetic radiation. We assumed a rectangular geometry with the period of $2 \mu \mathrm{m}$, in which single $\mathrm{Au}$ nanopillar resides on a $\mathrm{Au}$ substrate. We assumed the periodic boundary condition in the direction parallel to the substrate (Floquet condition). The incident beam direction was fixed at $60^{\circ}$ with respect to the direction normal to the substrate. We referred to Ref. 15 for the insertion of the dielectric function of $\mathrm{Au}$.

In Fig. 4, we show the result of the simulation at frequencies of $3.54 \times 10^{14} \mathrm{~Hz}$ (wavelength of $800 \mathrm{~nm}$ ) and 3.54 $\times 10^{12} \mathrm{~Hz}$ (wavelength of $80 \mu \mathrm{m}$ ) for a Au nanopillar with the diameter of $100 \mathrm{~nm}$ and the height of $2.4 \mu \mathrm{m}$. As is presented in Fig. 4(a), the distribution of electric field for the incident radiation at the frequency of $3.54 \times 10^{14} \mathrm{~Hz}$ exhibits a series of resonances along the nanopillar length. Consequently, the electric field at the tip $F_{\text {tip }}$ oscillates with increasing pillar height [Fig. 4(c)]. The ratio $F_{\text {tip }} / F_{\text {inc }}\left(F_{\text {inc }}\right.$ is the electric field of the incident radiation) reaches $\sim 10$ at the peak maximum of a narrow resonance for the pillar height of $\sim 0.8 \mu \mathrm{m}$. At the frequency of $3.54 \times 10^{12} \mathrm{~Hz}$, the $F_{\text {tip }} / F_{\text {inc }}$ ratio monotonously increases with the nanopillar height up to 30 .

\section{RESULTS AND DISCUSSION}

\section{A. Near infrared photocurrent}

In Fig. 5, we summarize the results of the near infrared photocurrent measurements for the five Au nanopillar samples, when the polarization of the laser pulses was within the incident plane (p-polarization). As the laser spot was moved across the sample from the peripheral flat $\mathrm{Au}$ area into the Au nanopillar domain, a sharp increase in the photocurrent was observed. The photocurrent of the Au nanopillars was distributed up to $\sim 50 \%$ as denoted in Fig. 5. We ascribe this to the spatial distribution of the CNT height over the template. Despite such signal distribution, it was clear that, for higher nanopillars, the photocurrent increases [Fig. 5(a)]. No

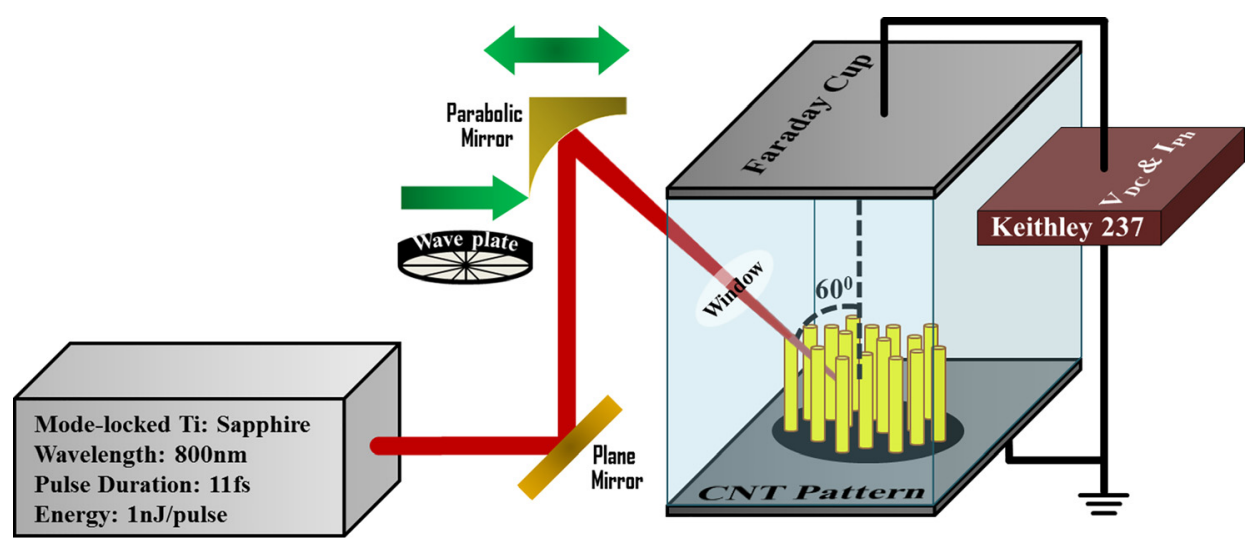

FIG. 3. (Color online) Schematic of the NIR photocurrent measurement setup. The NIR laser pulses produced from the mode-locked Ti:Sapphire laser is focused by a parabolic mirror on the $5 \mathrm{~mm}$-diameter Au nanopillar area of the sample with the spot size of $50 \times 100 \mu \mathrm{m}^{2}$. The irradiation position on the sample was shifted without any significant change of the laser spot size by moving the coupling and parabolic mirror simultaneously. 

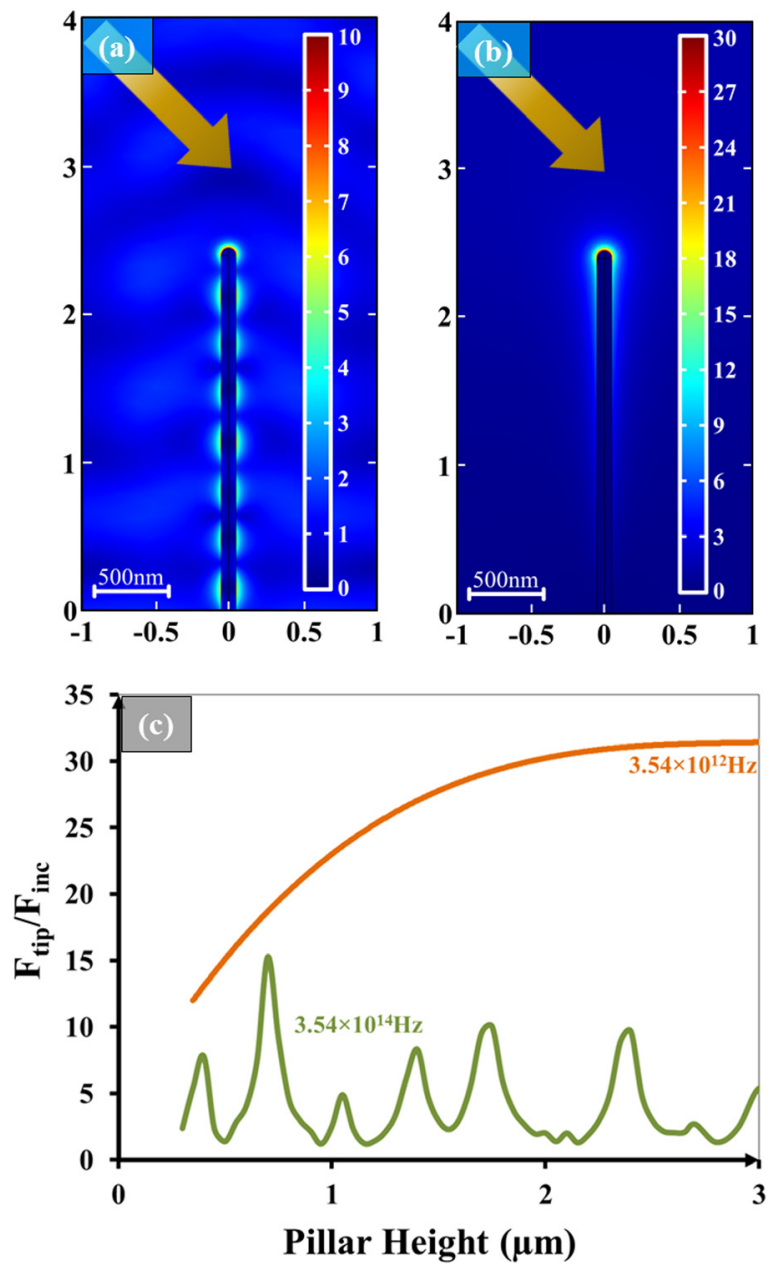

FIG. 4. (Color online) Electric field distribution of Au nanopillars $(2.4 \mu \mathrm{m}-$ height and $100 \mathrm{~nm}$-diameter) arrays when they are irradiated at the incident angle of $60^{\circ}$ (arrow in the figure) and p-polarized for the frequency (a) $3.54 \times 10^{14} \mathrm{~Hz}$ (the wavelength of $800 \mathrm{~nm}$ ) and (b) $3.54 \times 10^{12} \mathrm{~Hz}$ (the wavelength of $80 \mu \mathrm{m}$ ). The scales in (a) and (b) indicate the ratio $F_{\text {tip }} / F_{\text {inc }}$, where $F_{\text {tip }}$ is the electric field at the Au nanopillar tip apex and $F_{\text {inc }}$ is the electric field of the incident light. (c) The variation of the electric field $F_{\text {tip }}$ at the Au nanopillar tip apex with the increase in the Au nanopillar height for $3.54 \times 10^{14} \mathrm{~Hz}$ and $3.54 \times 10^{12} \mathrm{~Hz}$.

clear correlation observed between photocurrent and the $\mathrm{Au}$ nanopillar density as shown in Fig. 5(b). From this fact, unlike the recently reported photoinduced electron emission from dense CNT samples, ${ }^{16}$ we consider that thermal processes such as thermionic emission are not the origin of the observed current.

We ascribe the observed photocurrent as multiphoton photoemission with photon number $n$ in the range of 4-5, since the photon energy is equal to $\sim 1.6 \mathrm{eV}$ and the work function of the $\mathrm{Au}$ is equal to $\sim 5 \mathrm{eV}$. We found that the polarization dependence [Fig. 6] is consistent with this interpretation. In fact, as the polarization of the laser pulse was changed from p- $\left(0^{\circ}\right)$ to s-direction $\left(90^{\circ}\right)$, a steep reduction in the photocurrent was observed. We found that the observed angular dependence is proportional to $\left(F_{\text {tip }} / F_{\text {inc }}\right)^{2 n}$ with $n=4.5$, where $F_{\text {tip }}$ was calculated for different polarization angles for the nanopillar with the height of $2.7 \mu \mathrm{m}$. However, the experiment is not consistent with $n=1$ (single-

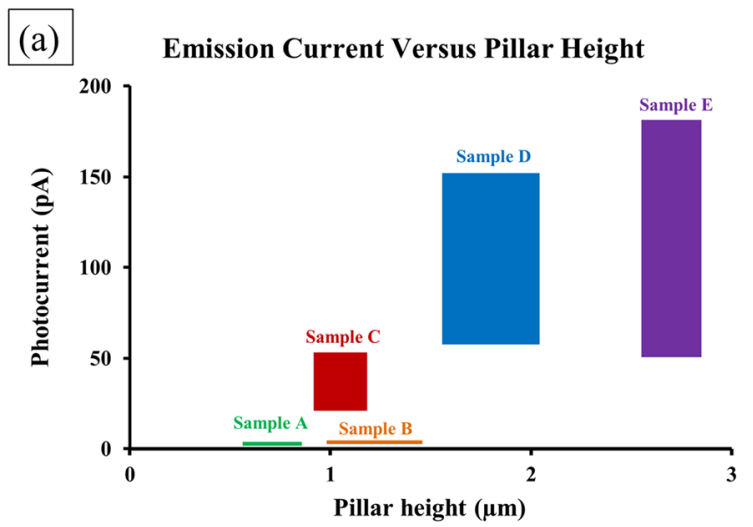

(b) Emission Current Versus Pillar Surface Density

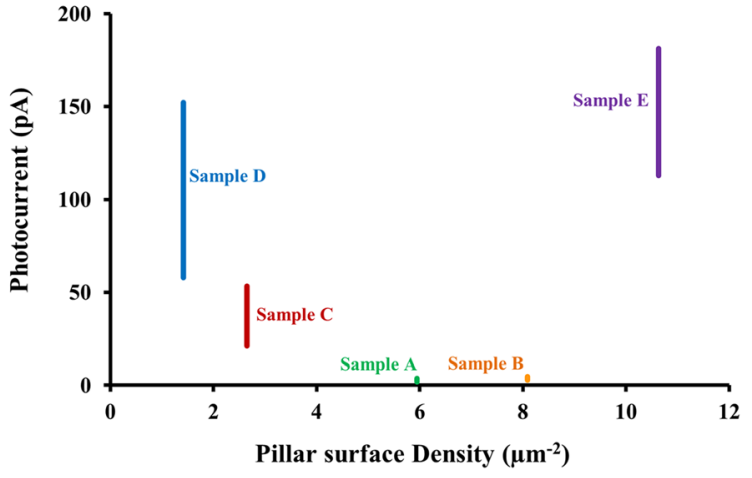

FIG. 5. (Color online) Relationship between the NIR photocurrent and (a) the Au nanopillar height, and (b) the Au nanopillar density.

photon photoemission case). We note that due to the additional dispersion of the wave plate, the peak electric field of the laser pulse was reduced by $\sim 30 \%$ without affecting the pulse energy. We also observed that the maximum photocurrent from the sample $\mathrm{E}$ was reduced from $\sim 180$ to $\sim 45 \mathrm{pA}$ after inserting the wave plate. This observation is compatible

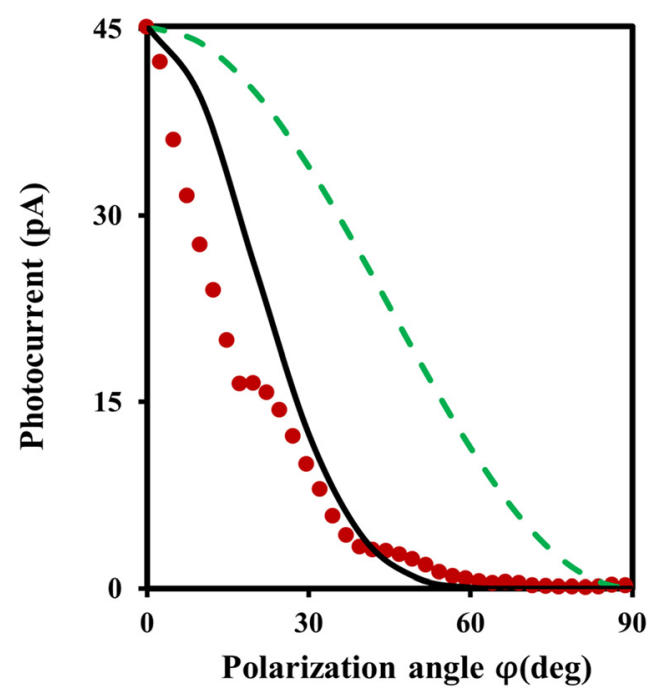

FIG. 6. (Color online) Polarization dependence of the NIR photocurrent of sample E (red circles). The solid curve shows the fit assuming the multiphoton photoemission process with the number of the photon $n$ equal to $4-5$. The single-photon process (dashed curve) is not compatible with the observation. 
with the decrease in electric field of the laser pulses due to the dispersion of the wave plate.

\section{B. Discussion}

The observed monotonous increase in the near infrared (NIR) photocurrent with the increase in the $\mathrm{Au}$ nanopillar height [Fig. 3(a)] is different from the oscillating behavior predicted by numerical simulation [Figs. 4(a) and 4(c)]. As the reason of this deviation, we consider the imperfections in the actual samples such as the distribution of the nanopillar height, the roughness [inset of Fig. 2(b)], the additional loss of the Au, and the loss caused by CNT as the core material. In the DC field emission case, in which the field enhancement of CNTs is dominated by the CNT height, it has been reported that only the tallest CNTs contribute to the emission. Since this is not the case at the optical frequency as suggested in Fig. 4(c), the height distribution of the nanopillar may smoothen out the height-induced resonance of the field enhancement. Both roughness and loss may cause the physical damping of the surface plasmon oscillation along the Au nanopillars. We indeed observed in SEM image of Fig. 2(b) that the Au nanopillar surface is not perfectly uniform. Additional simulations including such effects and experiments are needed to elucidate this.

Another interesting experiment is to test the $\mathrm{THz}$ response of these $\mathrm{Au}$ nanopillars, since the $\mathrm{THz}$ response might be insensitive to such surface roughness because of the longer wavelength. At $\mathrm{THz}$ frequencies, extremely high field enhancement up to $10^{4}$ has been reported for split-ring resonator structures. ${ }^{17,18}$ Therefore, the combination of the metal nanopillars fabricated by the method as proposed in the present work with a gate electrode having such resonant structure would open up an interesting field of research. We also foresee a development of $\mathrm{THz}$ field emission switches by controlling the spatial position and the shape of the grown CNTs. ${ }^{1,3,19,20}$ For example, fabrication of a CNT nanoswitch structure has been reported that operates by applying DC bias between a pair of spatially separated and shape controlled CNT forests to turn on the field emission. ${ }^{3}$ Such a nanoswitch structure coated by Au may operate efficiently at $\mathrm{THz}$ frequencies.

\section{SUMMARY}

Using carbon nanotube forests as template, we fabricated high-aspect ratio (up to 28) gold nanopillar cathodes. The irradiation of NIR short pulses produced photocurrent from the samples. The photocurrent increased with the increase in the nanopillar height, but it was uncorrelated with the density of the nanopillars. The observed photocurrent and its polarization dependence are compatible with the multiphoton photoemission. However, the exact origin of the NIR photoexcitation mechanism and the $\mathrm{THz}$ response of the $\mathrm{Au}$ nanopillars are the subjects of future study.

\section{ACKNOWLEDGMENTS}

The authors acknowledge the partial financial support from Iran National Science Foundation (INSF). Partial support from University of Tehran is also acknowledged. The authors also appreciate the support from Paul Scherrer Institute (PSI).

${ }^{1}$ Y. Abdi, J. Koohsorkhi, J. Derakhshandeh, S. Mohajerzadeha, H. Hoseinzadegan, M. D. Robertson, J. C. Bennettc, X. Wud, and H. Radamsona, Mater. Sci. Eng. C 26, 1219 (2005).

${ }^{2}$ L. C. Qin, D. Zhou, A. R. Krauss, and D. M. Gruen, Appl. Phys. Lett. 72, 3437 (1998).

${ }^{3}$ A. Sani, S. Darbari, Y. Abdi, and E. Arzi, Carbon 50, 3635 (2012).

${ }^{4}$ M. Monshipouri and Y. Abdi, J. Nanopart. Res. 17, 169 (2015).

${ }^{5}$ K. B. K. Teo et al., Nanotechnology 14, 204 (2003).

${ }^{6}$ K. B. K. Teo et al., Nature 437, 968 (2005).

${ }^{7}$ V. A. Guzenko, B. Pedrini, A. Menzel, and C. David, Microelectron. Eng. 121, 127 (2014).

${ }^{8}$ S. Gorelick, J. V. Comamala, V. A. Guzenko, and C. David, Microelectron. Eng. 88, 2259 (2011).

${ }^{9}$ Z. L. Wanga, M. B. Mohamed, S. Link, and M. A. El-Sayed, Surf. Sci. 440, L809 (1999).

${ }^{10}$ N. V. Hoang, S. Kumar, and G. H. Kim, Nanotechnology 20, 125607 (2009).

${ }^{11}$ H. Chen, L. Shao, Q. Li, and J. Wang, Chem. Soc. Rev. 42, 2679 (2013).

${ }^{12}$ S. L. Smitha, K. G. Gopchandran, T. R. Ravindran, and V. S. Prasad, Nanotechnology 22, 265705 (2011).

${ }^{13}$ Z. C. Xu, C. M. Shen, C. W. Xiao, T. Z. Yang, H. R. Zhang, J. Q. Li, H. L. Li, and H. J. Gao, Nanotechnology 18, 115608 (2007).

${ }^{14} \mathrm{X}$. Xu, Y. Zhao, X. Xue, S. Huo, F. Chen, G. Zou, and X. J. Liang, J. Mater. Chem. A 2, 3528 (2014).

${ }^{15}$ E. Palik, Handbook of Optical Constants of Solids (Academic, San Diego, CA, 1998).

${ }^{16}$ P. Yaghoobi, M. V. Moghaddam, and A. Nojeh, Solid State Commun. 151, 1105 (2011).

${ }^{17}$ M. Shalaby et al., Appl. Phys. Lett. 99, 041110 (2011).

${ }^{18}$ S. Bagiante, F. Enderli, J. Fabianska, H. Sigg, and T. Fuerrer, Sci. Rep. 5, 8051(2015).

${ }^{19}$ Y. Abdi, N. Otrooshi, and M. Miri, Curr. Appl. Phys. 14, 1287 (2014).

${ }^{20}$ S. Darbari, Y. Abdi, S. Mohajerzadeh, and E. Asl Soleimani, Carbon 48, 2493 (2010) 